\title{
Identification of Blood Serum Facial Markers and Their Crystallographic Features in Liver Pathologies
}

\author{
M.T. Botirov ${ }^{1 *}$, Yu.D.Khaitova ${ }^{2}$ \\ ${ }^{1}$ Fergana branch of the Tashkent Medical Academy Uzbekistan \\ ${ }^{2}$ Termez branch of the Tashkent Medical Academy Uzbekistan
}

DOI: $10.36348 /$ SJLS.2019.v04i09.007 $\quad$ | Received: 07.10.2019| Accepted: 14.10.2019| Published: 30.10 .2019

*Corresponding author: M.T. Botirov

\section{Abstract}

In this work, the facies of blood serum were studied in patients with liver pathologies and pathological markers were identified, and information on their crystallographic features was given. To solve the set tasks, wedge dehydration methods, computer morphometry, biochemical, microscopic and statistical research methods were used. The work studied the correlation between the features of the change from the norm, the magnitude of informative biochemical blood parameters of patients with established diagnoses of liver pathology, as well as the qualitative and quantitative characteristics of the morphotexture of the solid phase facies of their blood.

Keywords: biological fluid, facies, liver pathology, blood serum, crystallographic method.

\begin{abstract}
Copyright @ 2019: This is an open-access article distributed under the terms of the Creative Commons Attribution license which permits unrestricted use, distribution, and reproduction in any medium for non-commercial use (NonCommercial, or CC-BY-NC) provided the original author and source are credited.
\end{abstract}

\section{RELEVANCE OF THE WORK}

In the world today, the problem of liver diseases among various groups of the population remains as a result of viral diseases and diseases of the circulatory system, which is associated with an increase in their number and a high mortality rate [4]. According to the World Health Organization, the incidence of chronic viral hepatitis $\mathrm{B}$ and $\mathrm{C}$ rises day by day, more than 2 billion people in the world are infected with the hepatitis B virus, 300-400 million of them are chronic carriers of this infection, 130-170 million people are chronically infected with the hepatitis C virus [3]. Creating an effective system of prevention, diagnosis and treatment of the disease today is an important problem in medicine.

Scientific research is being conducted in the world aimed at the development of minimally invasive innovative technologies widely used as acute and chronic viral hepatitis, measures to prevent liver diseases, to develop methods for early diagnosis and effective methods of treating patients with liver diseases. Today, this problem is reasonably solved using the methods of biochemical and instrumental studies, in some cases even using histological methods. Despite this, their accuracy and informational content do not in all cases adequately reflect the true state of the pathology. In this case, the diagnosis of acute pathologies of viral hepatitis, if patients are in stationary conditions and receive full treatment, their condition can be considered satisfactory. In other circumstances, if the treatment was not carried out qualitatively, then the disease becomes chronic, fibrosis occurs in the form of a complication of the disease with the transition to cirrhosis, this is a serious actual problem, and this is proved by the fact that in the stage of fibrosis the disease in patients is not very symptomatic. Of particular importance is the development of methods for diagnosing and treating a disease to reduce complications, as well as improving measures for the occurrence of diseases associated with metabolism, as well as improving the quality of life of the population $[1,2]$.

\section{OBJECTIVE}

Improvement of the introduction of diagnostic markers of the pathological process based on the analysis of the relationship between clinical and biochemical and morphological indicators

\section{The object and methods of research}

To solve the set tasks, wedge dehydration methods, computer morphometry, biochemical, microscopic and statistical research methods were used.

To solve the tasks by the method of wedgeshaped dehydration, solid phases of blood serum were obtained in only 283 patients (male-137, female-146) at 
various ages and having viral liver diseases between the ages of 16-23 years. All of them were treated in the Fergana Oblast and Margilan City Infectious Diseases Hospital.

For crystallographic studies, samples of biological fluids were obtained from venous blood of the examined patients in a biochemical laboratory. From all studied patients, samples were received mainly 2 times - at the time of admission to the hospital and at discharge. In total, more than 600 samples of dehydrated blood serum of patients were prepared for study.

\section{RESULTS AND DISCUSSION}

In accordance with the goal and objectives, a correlation was studied between the features of the change from the norm, the magnitude of informative biochemical blood parameters of patients with established diagnoses of liver pathology, and the qualitative and quantitative characteristics of the morphotextura of the solid phase of their blood.

We studied the features of the manifestation in the morphology of the facies of the serum of patients, that is, their biochemical parameters, such as bilirubin, proteins of different fractions, AlT, AST and thymol test. These indicators, according to clinical biochemistry, are most sensitive to functional states of the liver and significantly change depending on changes in the liver, that is, they are highly informative biochemical markers of liver pathology.

We have investigated the quantitative morphometry of the systemic and subsystem structures of the facies and established the main types of the structural organization of blood serum in liver pathologies. Analysis of the crystallographic peculiar features of the blood serum (SC) allowed us to identify 4 types of its structural self-organization in liver pathologies: partially radial, irradial, circulatory, and "three-beam".

As a result of the analysis of the data obtained from the selected four types of structural organization of the SC, characteristic of liver disease was irradial, circulatory and "three-beam". In patients with acute viral hepatitis (AVH), chronic hepatitis (CG) and cirrhosis of the liver (CP), facies of the irradial type prevailed and this type became dominant. Much less frequently circulatory and three-radiated types were registered in $\mathrm{AVH}$ and $\mathrm{CG}$, and in $\mathrm{CP}$, circulatory as well as three-radial types were noted in episodic cases. A partially radial type of structure building of the UK facies was found only in the control group.

Thus, SC in patients with research using the method of wedge-shaped dehydration, having significant differences from the norm, is characterized by the presence of at least one of the main structural elements (large cracks, sectors, individualities or nodules).

In the following, we analyzed the qualitative and quantitative indicators of the facies of a dehydrated serum drop depending on the pathology of the liver and some of their biochemical parameters (Fig. 1).

In fig. 1 shows the formation of biochemical components of patients upon admission and discharge from the hospital in their dehydrated SC facies.

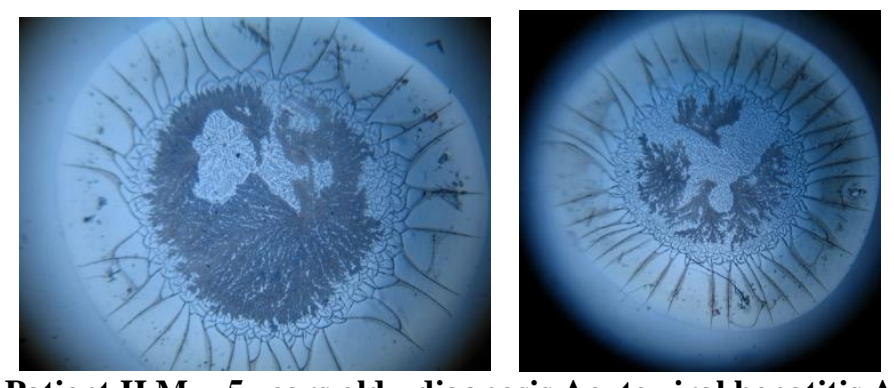

Patient H.M. - 5 years old - diagnosis Acute viral hepatitis A

\author{
Upon enrolment \\ ALT - 4.7, AST - 2.4 \\ Total bilirubin -130 \\ Free bilirubin - 100 \\ Bilirubin bound -30
}

\author{
At discharge \\ ALT -0.4 , AST -0.2 \\ Total bilirubin - 7
}




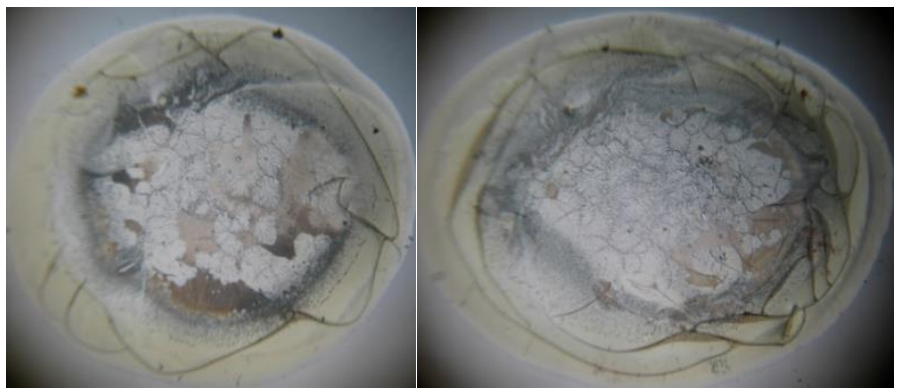

Patient B. M. - 22 years old - diagnosed with Chronic hepatitis B

\section{Upon enrolment}

ALT - 1.6, AST - 0.8

Total bilirubin -17

Free bilirubin - 10

Bilirubin bound - 7
At discharge

ALT -0.8 , AST -0.5

Total bilirubin -7

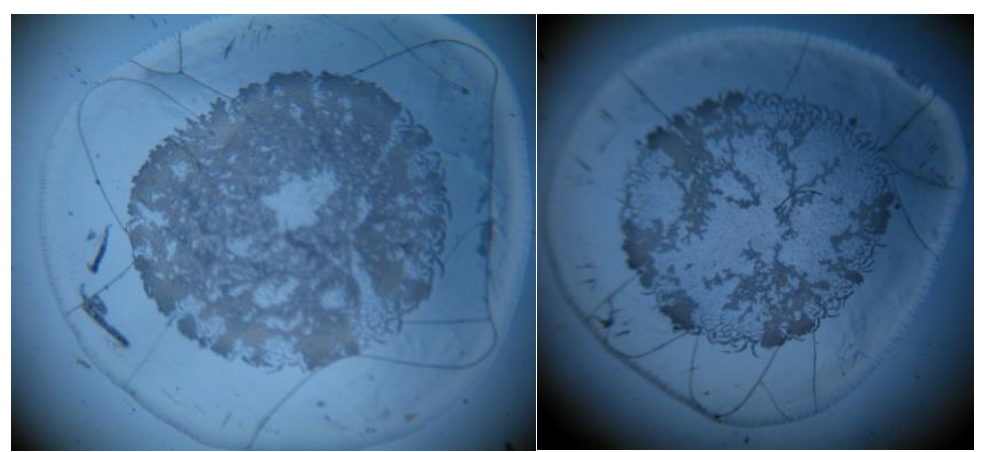

Patient K.I. - 28 years old - diagnosis Chronic hepatitis C

Upon enrolment

ALT - 4.7

AST -2.4

Total bilirubin -165

Free bilirubin - 120

Bilirubin bound -45

\section{At discharge}

ALT - 0.4

AST -0.2

Total bilirubin - 7

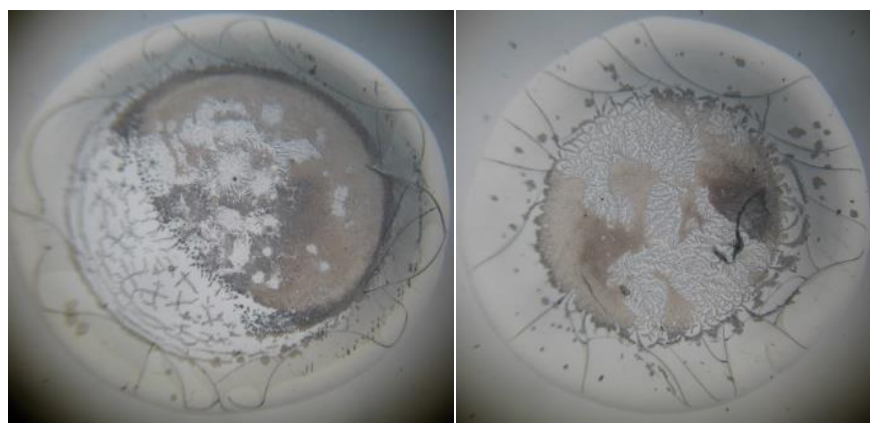

Patient H.N. - 42 years old - diagnosis of liver cirrhosis in the stage of decompensation

\section{Upon enrolment}

ALT - 0.6, AST - 0.3

Total bilirubin - 93

Free bilirubin - 54

Bilirubin bound - 39
At discharge

$$
\begin{aligned}
& \text { ALT }-0.4 \text {, AST }-0.2 \\
& \text { Total bilirubin - } 17 \\
& \text { Free bilirubin }-10 \\
& \text { Bilirubin bound }-7
\end{aligned}
$$

Fig-1: Analysis of qualitative and quantitative indicators of the facies of dehydrated serum drop depending on the pathology of the liver and some of their biochemical parameters

Analysis of the data made it possible to identify the most characteristic and frequently encountered abnormal structures in liver cirrhosis, which include leaf-shaped structures and various types 
of cracks, concentration free, etc. The main distinguishing feature of the facies of blood serum in liver diseases was the polymorphism of pathologically modified cracks. In the facies, bundle blocks, "bundle" structures in the central zone and periphery, circulatory cracks were observed.

In the structure of the facies, markers of stagnation - three-beam cracks, leaf-shaped structures were signs of the sclerotherapy process, dominated the most frequently encountered local violations of the structural organization of SC in liver cirrhosis.

According to the results of studying the structure of the facies, it was established that leaf-like structures were significantly more common in liver cirrhosis than in chronic hepatitis, and the "Moss" figure, rounded inclusions and circular cracks are recorded with approximately the same frequency.

We know that the level of structural construction of the SC reflects the degree of tension of the adaptation systems of the body: the more pronounced adaptation reactions, the higher the activity of structure formation.

\section{CONCLUSIONS}

The main crystallographic features of blood serum in acute, chronic hepatitis and cirrhosis of the liver according to the method of wedge-shaped dehydration have been established, 3 types of its structural organization have been identified: irradial, circulatory, "three-beam". In acute, chronic hepatitis and cirrhosis of the liver, irradial-type facies prevailed, the percentage of which in patients with liver cirrhosis was almost 1.5 times higher. In acute hepatitis, facies with a "three-beam", circulatory type was observed, in chronic hepatitis, a circulatory type of structure was observed. In the blood serum of healthy people, $100 \%$ of cases of partially radial type of structure were observed.

It was revealed that the most frequent anomalous structural elements of the facies in liver cirrhosis were leaf-shaped, cracks of various types, especially "three-beam" cracks, concentration waves, which are a sign of the sclerotherapy process and markers of stagnation. In acute and chronic hepatitis, circulatory fissures have been identified, "harness" structures in the central zone and periphery.

\section{REFERENCES}

1. Turgunboyevich, B. M. (2016). Some results of experiments to study the morphology of the solid phase dehydrated biological fluids of healthy and sick people with diseases of liver. Austrian Journal of Technical and Natural Sciences, (1-2).

2. Botirov M.T. (2018). Morphological Indicators of the Facies of Biological Fluids in Patients with Liver Diseases // International Journal of Biology, 10(2).

3. Hatzakis, A., Wait, S., Bruix, J., Buti, M., Carballo, M., Cavaleri, M., ... \& Esteban, R. (2011). The state of hepatitis B and $\mathrm{C}$ in Europe: report from the hepatitis B and C summit conference. Journal of Viral Hepatitis, 18, 1-16.

4. Komarov, F. I., Osadchuk, M. A., \& Osadchuk, A. M. (2010). Practical gastroenterology. Moscow, MIA. 